\title{
THE URANUS OCCULTATION OF 10 JUNE 1979. I. THE RINGS
}

\section{P. D. NiCHOLSON}

Mount Stromlo and Siding Spring Observatories, Research School of Physical Sciences, Australian National University, Woden P.O.,

Canberra, Australia 2606

\author{
K. MatThews \\ California Institute of Technology, Pasadena, California 91125
}

\section{P. GOLDREICH}

Division of Geological and Planetary Sciences, California Institute of Technology, Pasadena, California 91125

\author{
Received 2 October 1980
}

\begin{abstract}
Observations and analysis of a stellar occultation by the rings of Uranus on 10 June 1979 are presented. Occultations by rings $4, \alpha, \beta, \gamma, \delta$, and $\epsilon$ are identified, and radii and azimuths of the occulting segments in the plane of the rings calculated. Results for rings $\gamma$ and $\delta$ are consistent with the hypothesis (Elliot et al. 1978; Nicholson et al. 1978) that these two rings are circular and coplanar, and an approximate upper limit of $8 \times 10^{-5}$ is placed on the eccentricity of either ring. Coplanar elliptical models are presented for rings $\alpha$ and $\beta$, with eccentricities of $(6.0 \pm 0.3) \times 10^{-4}$ and $(4.9 \pm 0.5) \times 10^{-4}$, respectively. For ring 4 two possible elliptical models are obtained, with eccentricities of $(1.2 \pm 0.4) \times 10^{-3}$ and $(6.0 \pm 0.3) \times 10^{-4}$, the former being preferred. The width-radius relation established previously for the $\epsilon$ ring is confirmed, and the elliptical model for this ring is slightly revised. An improved estimate for Uranus's $J_{2}$ of $(3.390 \pm 0.005) \times 10^{-3}$, based on the apsidal precession of the $\epsilon$ ring, and an upper limit for $\left|J_{4}\right|$ of $\sim 1 \times 10^{-4}$, based on the precession of rings 4 and $\beta$, are obtained.
\end{abstract}

\section{INTRODUCTION}

On 10 June 1979 a stellar occultation by Uranus and its ring system, predicted by Klemola and Marsden (1977), was observed at Las Campanas Observatory in Chile. Data from the ring occultations, and their analysis, are presented in this paper, while an analysis of the planetary occultation is to be presented in a subsequent paper.

Previous occultations by the rings of Uranus have been observed on 10 March 1977 (Elliot et al. 1978 and references therein), 23 December 1977 (Millis and Wasserman 1978), and 10 April 1978 (Nicholson et al. 1978), from which a fairly detailed picture of the ring system has emerged. All nine of the rings so far identified $(6,5,4, \alpha, \beta, \eta, \gamma, \delta$, and $\epsilon$, in order of increasing radius) appear to lie in, or very close to, the common orbital plane of Uranus's satellites, and uncertainty in the orientation of this plane dominates the present uncertainties in the absolute radii of the rings (Elliot et al. 1978). Radial widths of the rings range from $\lesssim 5 \mathrm{~km}(6$, $5,4, \gamma$, and $\delta$ ) to $100 \mathrm{~km}$ (widest part of $\epsilon$ ), with optical depths being of the order of unity or greater (Nicholson et al. 1978). A linear relation between radial width and apparent radius for the $\epsilon$ ring led Nicholson et al. to propose an elliptical model for this ring, which involved a determination of the rate of apsidal precession due to Uranus's oblate figure.

\section{OBSERVATIONS}

Observations of the occultation were made with an infrared photometer mounted on the $2.5-\mathrm{m}$ du Pont telescope at Las Campanas Observatory in Chile. A refinement of the $\epsilon$ model, as well as new elliptical models for rings $4, \alpha$, and $\beta$. Departures of these three rings from circularity have previously been suspected, but there have been insufficient data to completely constrain models (Elliot et al. 1978; Nicholson et al. 1978). In addition, these observations of the ring occultations serve to locate the center of mass of Uranus, relative to the apparent track of the occulted star on the sky. This is an essential prerequisite to the analysis of the planetary occultation.

Details of the observational procedure are given in Sec. II, and the data are presented in Sec. III. In Sec. IV, results of the data analysis are presented, which are combined in Sec. V with the results of previous occultations to produce models for rings $4, \alpha, \beta$, and $\epsilon$. Section VI contains a discussion of Uranus's gravitational moments, $J_{2}$ and $J_{4}$, and our conclusions are presented in Sec. VII. In the Appendix, results of a reanalysis of the 10 April 1978 occultation, in which certain small inconsistencies with the present work are removed, are given.

(C) 1981 Am. Astron. Soc. 
TABLE I. Ring occultation observations

\begin{tabular}{|c|c|c|c|c|c|c|c|c|c|c|}
\hline \multirow{3}{*}{$\begin{array}{l}\text { Ring } \\
\text { identi- } \\
\text { fication }\end{array}$} & \multicolumn{5}{|c|}{ Immersion } & \multicolumn{5}{|c|}{ Emersion } \\
\hline & & UTC & & Duration $^{a}$ & Fractional & & UTC & & Duration $^{a}$ & Fractional \\
\hline & $\mathrm{h}$ & $\mathrm{m}$ & $\mathrm{s}$ & (s) & $\operatorname{depth}^{\mathrm{b}}$ & $\mathrm{h}$ & $\mathrm{m}$ & $\mathrm{s}$ & (s) & depth $^{b}$ \\
\hline 4 & & - & & - & - & 02 & 47 & 17.0 & 0.3 & 0.4 \\
\hline$\alpha$ & 01 & 40 & 30.2 & 0.3 & 0.7 & 02 & 48 & 59.7 & 0.6 & 0.6 \\
\hline$\beta$ & 01 & 39 & 44.7 & 0.3 & 0.5 & 02 & 49 & 42.8 & 0.3 & 0.5 \\
\hline$\gamma$ & 01 & 38 & 11.8 & 0.2 & 0.5 & 02 & 51 & 15.4 & 0.2 & 0.4 \\
\hline$\delta$ & 01 & 37 & 40.2 & 0.2 & 0.4 & 02 & 51 & 47.3 & 0.2 & 0.6 \\
\hline$\epsilon$ & 01 & 35 & 17.4 & 3.55 & $0.4-0.9$ & 02 & 53 & 56.9 & 2.4 & $0.5-0.9$ \\
\hline
\end{tabular}

a FWHM.

b Not corrected for planetary and ring flux (see text).

standard $K$ filter $\left(\lambda_{0}=2.20 \mu \mathrm{m}, \Delta \lambda=0.4 \mu \mathrm{m}\right)$ was used, with an InSb detector cooled to $55 \mathrm{~K}$. The observational procedure was essentially the same as that described by Nicholson et al. (1978) for the occultation of 10 April 1978, except that the reference position used for sky subtraction was 30 arcsec south of the star, and that the focal plane aperture was 5 arcsec in diameter (centered on the star). Output from the photometer was recorded on a strip chart, with timing marks at intervals of $1 \mathrm{~s}$ obtained from a WWV receiver. The time constant of this recording system was $0.1 \mathrm{~s}$, and the absolute timing accuracy was $\pm \lessgtr 0.3 \mathrm{~s}$.

The signal-to-noise ratio of the data, for the star unocculted, is $\sim 5$, and the contribution by the planet and its rings to the $2.2-\mu \mathrm{m}$ flux entering the 5 -arcsec diameter aperture varied from $\sim 15 \%$ at the times of the $\epsilon$-ring occultations to $18 \%$ at the time of planetary immersion and $32 \%$ at planetary emersion. These figures are consistent, given small guiding and seeing variations, with a $K$ magnitude of $\sim 11.5$ for the occulted star, estimated from values of $V$ and $B-V$ given by Liller (1977). A rough measurement made 30 min before the first $\epsilon$-ring occultation indicated that $K(\mathrm{star}) \sim 11.2$.

Continuous observations commenced at $\sim 1: 16$ UTC, 10 June 1979, and the first occultation by the $\epsilon$ ring occurred at 1:35:17 UTC, only three minutes ahead of Klemola and Marsden's (1977) prediction. At 1:52:30 UTC, the onset of planetary occultation was observed, following which observations were suspended from $\sim 1: 55$ to $\sim 2: 26$ UTC. The star emerged from planetary occultation at 2:37:40 UTC, and the second $\epsilon$-ring occultation occurred at 2:53:57 UTC, two minutes ahead of the predicted time. Observations ceased at $\sim 2: 56$ UTC.

\section{RING OCCULTATIONS}

Careful examination of the recordings revealed 11 identifiable ring occultations, five preceding and six following the planetary occultation. These events were subsequently identified with rings $\alpha, \beta, \gamma, \delta$, and $\epsilon$ (immersion and emersion), and ring 4 (emersion only). Several additional brief spikes in the recordings correlate neither with one another nor with any of the known rings, and will, henceforth, be disregarded. Failure to detect occultations by rings $\eta, 5,6$, and 4 (immersion) is consistent with the relatively low signal-to-noise ratio of the present data, compared with that obtained from previous occultations.

In Table I are listed the central times (UTC), durations (full width at half maximum extinction), and fractional depths of the identified ring occultations. Uncertainties in the central times are $\pm 0.1 \mathrm{~s}$, except for the $\alpha$ emersion event, for which the uncertainty is $\pm \sim 0.2$ $\mathrm{s}$. Absolute timing accuracy is $\pm 0.3 \mathrm{~s}$. Except for the $\epsilon$ occultation, the durations are significantly influenced by both the 0.1 -s system time constant and noise. Fractional depths are expressed relative to zero total flux, and have not been corrected for the variable and somewhat uncertain planetary and ring contributions.

Segments of the strip chart recordings are reproduced in Fig. 1. As in previous occultation observations, only the occultation profiles for the $\epsilon$ ring exhibit any resolved structure. This structure is quite similar to that reported in 1977 by Millis et al. (1977) and in 1978 by Nicholson et al. (1978).

\section{ANALYSIS}

\section{a) Ring Geometry}

The positions of the occulting segments of the rings, projected on the plane of the sky, are calculated from the observed occultation times in the manner described by Elliot et al. (1978). This procedure is a modification of Bessel's method for the investigation of lunar occultations (see Smart 1977), and necessitates calculation of the apparent geocentric positions of star and planet, as well as the geocentric coordinates of the observing site, separately for each occultation event. The resulting positions are expressed as displacements of the occulting material to the east $(\xi)$ and north $(\eta)$, in the plane of the sky, from the instantaneous ephemeris position of the center of the planet. Numerical values for the various parameters used in the reduction, and for some others to be used subsequently, are given in Table II. The apparent geocentric position of Uranus is obtained by interpolation in the Astronomical Ephemeris (AE) tabulation of daily positions, which incorporate corrections for light-travel time. Stellar coordinates for the epoch 1950.0 are taken from Klemola and Marsden (1977). 

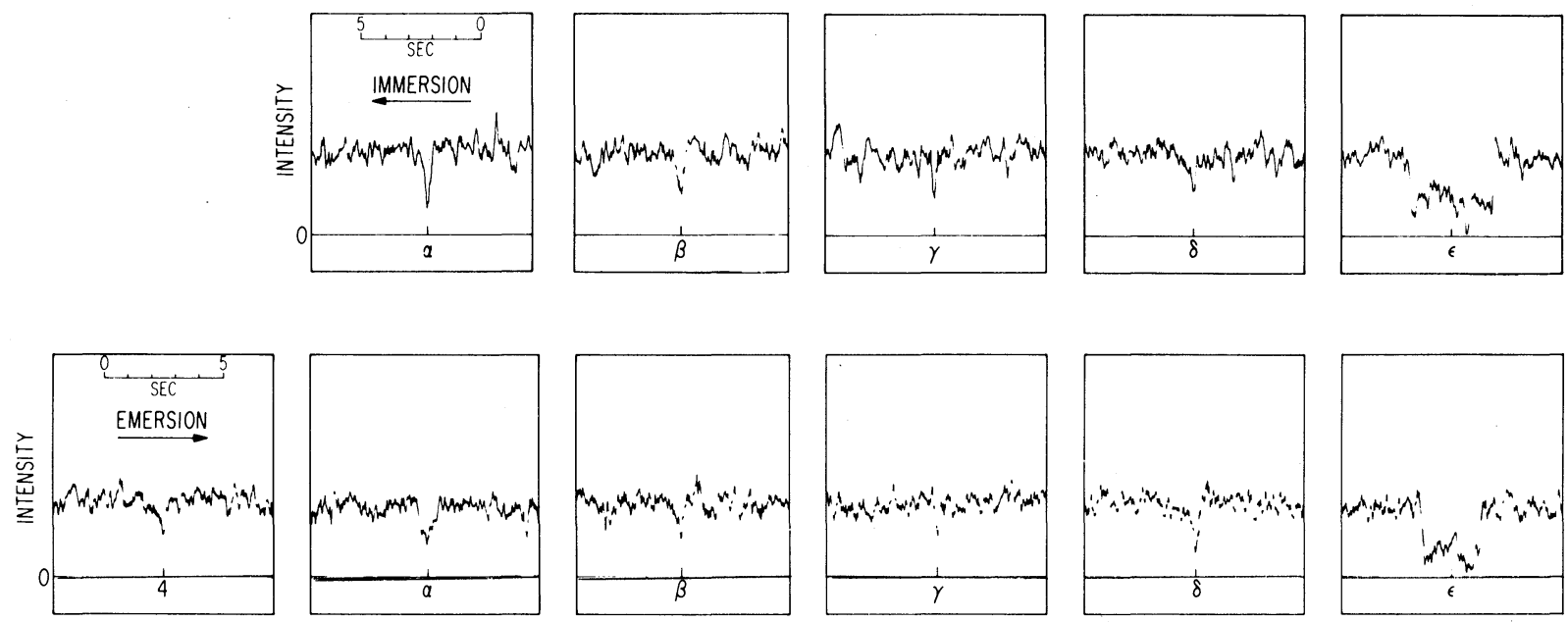

FIG. 1. Excerpts from the strip chart recording of the 10 June 1979 occultation, showing all identified ring occultations. The time axis of the emersion records has been reversed, so that they may be more readily compared with the immersion records above them, although no attempt has been made at precise alignment. Occultation times, as indicated by the tick marks, are given in Table I.

Positions for the 11 ring segments are listed in Table III. They have been corrected for general relativistic deflection of the light beam by Uranus, and for a constant offset in the center of Uranus from its ephemeris position of

$$
\Delta \xi=-3578.9 \mathrm{~km}
$$

and

$$
\Delta \eta=-1804.6 \mathrm{~km} \text {. }
$$

This offset, equivalent to $-0.277 \mathrm{arcsec}$ in right ascension and -0.140 arcsec in declination, accounts for errors in both the ephemeris of Uranus and the stellar position. Its determination is considered below.

To transform from the above "sky plane coordinates" to polar coordinates in the plane of the rings, the orien-

TABLE II. Parameters used in the 10 June 1979 occultation

\begin{tabular}{|c|c|}
\hline Parameter & Value \\
\hline $\begin{array}{l}\text { Geocentric coordinates of observatory } \\
\text { Radius }(\mathrm{km}) \\
\text { Latitude } \\
\text { West longitude } \\
\text { Greenwich sidereal time at } 0^{\mathrm{h}} \mathrm{ET}^{\mathrm{a}} \\
\Delta T=\mathrm{ET}-\mathrm{UT}^{\mathrm{a}}(\mathrm{s}) \\
\text { Astronomical unit } \\
\mathrm{Jul}^{\mathrm{a}}(\mathrm{km}) \\
\text { Elements of satellites' orbital plane } \\
\quad \text { (equinox of date): } \\
J \\
N \\
\text { Auxiliary angles } \mathrm{c}: \\
B \\
P \\
U\end{array}$ & $\begin{array}{l}6375.447 \\
-28^{\circ} .84075 \\
70^{\circ} .70195834 \\
17^{\mathrm{h}} 10^{\mathrm{m}} 10^{\mathrm{s} 9} \\
50.0 \\
1.496 \times 10^{8} \\
2444034.5\end{array}$ \\
\hline
\end{tabular}
analysis.

a Astronomical Ephemeris (AE) for 1979.

b Dunham (1971).

c See Explanatory Supplement to the AE, 1961, p. 342 for definitions. tation in space of the ring plane must be specified. (Observations of this same occultation from several sites on the Earth might have permitted the independent determination of the plane's orientation, but such is not presently the situation.) Adopting the common orbital plane of Uranus's four outer satellites, determined by Dunham (1971), as a reference plane, Elliot et al. (1978) found that the average inclination of rings $\alpha, \beta, \gamma$, and $\delta$ was $0^{\circ} .7 \pm 2^{\circ}$. 1 , i.e., indistinguishable from zero. Individual determinations of the inclinations of rings $\gamma$ and $\delta$, the only rings that Elliot $e$ al. found to be circular and concentric, yielded upper limits of 0.08 and 0.13 , respectively. Variations in the apparent radius and width of the $\epsilon$ ring were shown by Nicholson et al. (1978) to be consistent with an eccentric ring of zero inclination, rather than with a circular inclined ring. It is therefore assumed that all of the rings are coplanar, and that they lie in the common orbital plane of the satellites, as determined by Dunham (1971).

In the absence of available observations of the occultation from other sites, it is necessary to make some further $a$ priori assumptions concerning the dimensions of the ring system in order to determine the appropriate apparent offset of the center of mass of Uranus from its

TABLE III. Sky-plane coordinates of the occulting segments of the

\begin{tabular}{|c|c|c|c|c|}
\hline \multirow[b]{2}{*}{ Ring } & \multicolumn{2}{|c|}{ Immersion } & \multicolumn{2}{|c|}{ Emersion } \\
\hline & $\overline{\xi-\Delta \xi^{\mathrm{a}}}$ & $\eta-\Delta \eta^{\mathrm{b}}$ & $\xi-\Delta \xi^{\mathrm{a}}$ & $\eta-\Delta \eta^{b}$ \\
\hline 4 & - & - & 36694 & -2495 \\
\hline$\alpha$ & -35513 & 17281 & 38542 & -3003 \\
\hline$\beta$ & -36332 & 17505 & 39318 & -3217 \\
\hline$\gamma$ & -38005 & 17961 & 40985 & -3675 \\
\hline$\delta$ & -38574 & 18116 & 41559 & -3833 \\
\hline$\epsilon$ & -41145 & 18818 & 43892 & -4475 \\
\hline
\end{tabular}
rings, in kilometers.

a $\Delta \xi=-3578.9$.

${ }^{\mathrm{b}} \Delta \eta=-1804.6$ 
ephemeris position. From multiple observations of the occultation of SAO 158687 on 10 March 1977, Elliot et al. (1978) obtained a simultaneous least-squares solution for the radii of rings $\alpha, \beta, \gamma$, and $\delta$ (solution 2). It was assumed that all four rings are circular, are concentric, and lie in the orbital plane of Uranus's four outer satellites. Although systematic departures from this solution were noted for rings $\alpha$ and $\beta$, the observations of $\gamma$ and $\delta$ were found to be quite consistent with this simple model, with radii of $47746 \pm 27$ and $48423 \pm 27 \mathrm{~km}$, respectively. Individual solutions for the radius and center of each of these two rings gave slightly larger radii of $47755 \pm 18$ and $48441 \pm 26 \mathrm{~km}$, respectively, and centers separated by an insignificant $5 \pm 17 \mathrm{~km}$ in $\xi$ and $15 \pm 54 \mathrm{~km}$ in $\eta$.

To determine the offset $(\Delta \xi, \Delta \eta)$, therefore, it is assumed here that the $\gamma$ and $\delta$ rings are circular and concentric, and that their radii are exactly 47746 and 48423 $\mathrm{km}$. Since the observations of only one such circular ring of known radius suffice to determine the offset, a check on the consistency of our assumptions is provided by observations of the second ring. For the $\gamma$ ring, it is found that

$$
(\Delta \xi, \Delta \eta)_{\gamma}=(-3578.8 \pm 2.4,-1796.9 \pm 9.5),
$$

while for the $\delta$ ring,

$$
(\Delta \xi, \Delta \eta)_{\delta}=(-3579.1 \pm 2.4,-1812.4 \pm 9.5),
$$

with all dimensions in kilometers. These two determinations are consistent within the stated uncertainties, which reflect the 0.1 -s timing uncertainties in the data, and their mean is adopted as the best estimate of the offset. It should be noted that this consistency, while lending support to the hypothesis that the $\gamma$ and $\delta$ rings are circular and concentric, does not necessarily imply that the absolute values of the adopted radii are correct. As long as the radius difference of $\sim 677 \mathrm{~km}$ is maintained, considerable changes in the radii could readily be accommodated by a change in the offset.

Transformation of the corrected sky-plane coordinates, $(\xi-\Delta \xi, \eta-\Delta \eta)$, of the occulting ring segments to polar coordinates in the ring plane is straightforward, and involves the standard auxiliary angles, $B, P$, and $U$, derived from the elements of the satellites' orbital plane (see Table II). Table IV presents the results. The azimuth, $\theta$, is measured in the direction of the satellites' orbital motion from the ascending node of the ring plane on the Earth's equator of date.

The ranges of radius $R$ spanned by the current observations are $30000-76200 \mathrm{~km}$ and $30900-53800 \mathrm{~km}$ for immersion and emersion, respectively, the lower limits being set by the interception of the light beam by the planet's atmosphere.

It is emphasized that, while the analysis of the ring occultations presented here is consistent with that carried out by Elliot et al. (1978) for the 10 March 1977 occultation, particularly with respect to the adopted ring plane and the radii of $\gamma$ and $\delta$, the uncertainties in $R$ and
TABLE IV. Ring-plane coordinates of the occulting segments,

\begin{tabular}{|c|c|c|c|c|}
\hline \multirow[b]{2}{*}{ Ring } & \multicolumn{2}{|c|}{ Immersion } & \multicolumn{2}{|c|}{ Emersion } \\
\hline & $\overline{R(\mathrm{~km})^{\mathrm{a}}}$ & $\theta(\operatorname{deg})^{b}$ & $R(\mathrm{~km})^{\mathrm{a}}$ & $\theta(\operatorname{deg})^{b}$ \\
\hline 4 & - & - & 42687 & 174.604 \\
\hline$\alpha$ & 44811 & 13.939 & 44864 & 175.092 \\
\hline$\beta$ & 45776 & 13.737 & 45779 & 175.283 \\
\hline$\gamma$ & 47749 & 13.351 & 47746 & 175.668 \\
\hline$\delta$ & 48420 & 13.227 & 48424 & 175.794 \\
\hline$\epsilon$ & 51457 & 12.706 & 51180 & 176.270 \\
\hline
\end{tabular}
10 June 1979.

a $\pm 2 \mathrm{~km}$.

$\mathrm{b} \pm 0^{\circ} 001$.

$\theta$ given in Table IV reflect only the timing uncertainties $(0.1 \mathrm{~s})$ in the data. An error in the orientation of the ring plane, for example, could lead to systematic errors in all of the ring radii and azimuths much larger than those due to timing.

\section{b) Ring Widths}

Durations of the ring occultations, given in Table I, may be converted to apparent radial widths by multiplying them by the topocentric velocity of the star relative to Uranus, projected onto the radial direction, of $\sim 21.25 \mathrm{~km} \mathrm{~s}^{-1}$ for both immersion and emersion. For all the rings except $\epsilon$, however, these apparent widths are severely affected by (1) the time resolution of $0.1 \mathrm{~s}$, equivalent to $\sim 2 \mathrm{~km}$; (2) diffraction, with a Fresnel scale at $2.2 \mu \mathrm{m}$ of $\sim 2.4 \mathrm{~km}$; and (3) the angular diameter of the occulted star, equivalent to $\sim 0.4 \mathrm{~km}$ at the distance of Uranus.

Because of these effects, and because of the comparatively low signal-to-noise ratio of the present data, no improvement is possible on the estimates of radial widths and optical depths made by Nicholson et al. (1978) for the narrow rings.

For the $\epsilon$ ring, apparent radial widths, corresponding to the immersion and emersion occultations, are respectively 75 and $51 \mathrm{~km}$. These widths are not significantly affected by any of (1)-(3) above. Estimated opacities for this ring are quite sensitive to the amount of planetary and ring contribution to the total observed flux, but are $\sim 1.0$ (immersion) and $\sim 1.5$ (emersion), consistent with the conclusion of Elliot et al. (1978) that the opacity varies inversely with the radial width.

The radial widths and radii of the segments of the $\epsilon$ ring probed in this occultation are in accord with the width-radius relation established by Nicholson et al. (1978). In Table V are collected all available independent measurements of these two quantities. The several $\epsilon$ occultations observed on 10 March 1977, and uniformly reduced by Elliot et al. (1978), have been combined into two normal points with appropriate standard deviations. The estimated duration of the 10 April 1978 $\epsilon$ emersion occultation has been revised from 4.0 to 4.1 $\mathrm{s}$, and the radii for both immersion and emersion segments slightly decreased, as described in Sec. V a.

A weighted least-squares fit of the relation 
TABLE V. Radii and widths of the $\epsilon$ ring.

\begin{tabular}{ccccc}
\hline $\begin{array}{c}\text { Date of } \\
\text { observation }\end{array}$ & $\begin{array}{c}\text { Radius } \\
(\mathrm{km})\end{array}$ & \multicolumn{2}{c}{ Radial width $(\mathrm{km})$} \\
\cline { 3 - 5 } Observed & Model \\
\hline 10 Mar. 1977 a & 51702.5 & $98.0 \pm 4$ & 99.4 \\
10 Jun. 1979 & 51457 & $75 \pm 1$ & 76.4 \\
10 Apr. 1978 & 51434 & $76 \pm 1$ & 74.3 \\
23 Dec. 1977 & $\sim 51190$ & $47 \pm 3$ & 51.5 \\
10 Jun. 1979 & 51180 & $51 \pm 1$ & 50.5 \\
10 Mar. 1977 & 51054.5 & $38.5 \pm 1$ & 38.8 \\
10 Apr. 1978 & 50874 & $22 \pm 1$ & 21.9 \\
\hline \hline
\end{tabular}

a Elliot et al. (1978), average of several observations.

b This work.

c Nicholson et al. (1978), as revised here.

d Millis and Wasserman (1978).

$$
W=W_{0}+A\left(R-R_{0}\right)
$$

to these seven points, with $R_{0}=51269.4 \mathrm{~km}$ (see below), yields the result

$$
\begin{aligned}
& W_{0}=58.9 \pm 0.5 \mathrm{~km}, \\
& A=0.0935 \pm 0.0019 .
\end{aligned}
$$

Both the observations and the fitted relation are shown in Fig. 2, and the radial widths calculated from the above expression are also given in Table $\mathrm{V}$.

This systematic variation of radial width with radius is strong evidence for the elliptical nature of the $\epsilon$ ring (Nicholson et al. 1978), and may be interpreted in terms of confocal ellipses forming the inner and outer edges of the ring. If the semimajor axes and eccentricities of these bounding ellipses are denoted $a \pm \Delta a$ and $e \pm \Delta e$, respectively, where $a$ and $e$ are obtained from the elliptical model of the $\epsilon$ ring $\left(\epsilon_{9}\right)$ presented in Sec. V, then, with

$$
R_{0} \equiv a\left(1-e^{2}\right)
$$

we have

$$
W_{0}=2 \Delta a-4 e a \Delta e+O\left(e^{2} \Delta a\right)
$$

and

$$
A=2 \Delta e / e+2 \Delta a / a+O(e \Delta e) .
$$

From the above values of $W_{0}$ and $A$, we obtain

$$
\begin{gathered}
\Delta a=29.7 \pm 0.3 \mathrm{~km}, \\
\Delta e=(3.66 \pm 0.09) \times 10^{-4} .
\end{gathered}
$$

Note that the dimensionless eccentricity gradient across the ring, $a \Delta e / \Delta a(=0.632)$, is of order unity, as pointed out by Dermott et al. (1979).

The dominant second-order correction to Eq. (1) for elliptical ring boundaries is

$$
\Delta e /(e a)\left(R-R_{0}\right)^{2},
$$

and amounts to no more than $0.15 \mathrm{~km}$ for the $\epsilon$ ring.

V. MODELS OF RINGS $4, \alpha, \beta$, AND $\epsilon$

a) A Homogeneous Data Set

Before the attempt is made to construct models for any

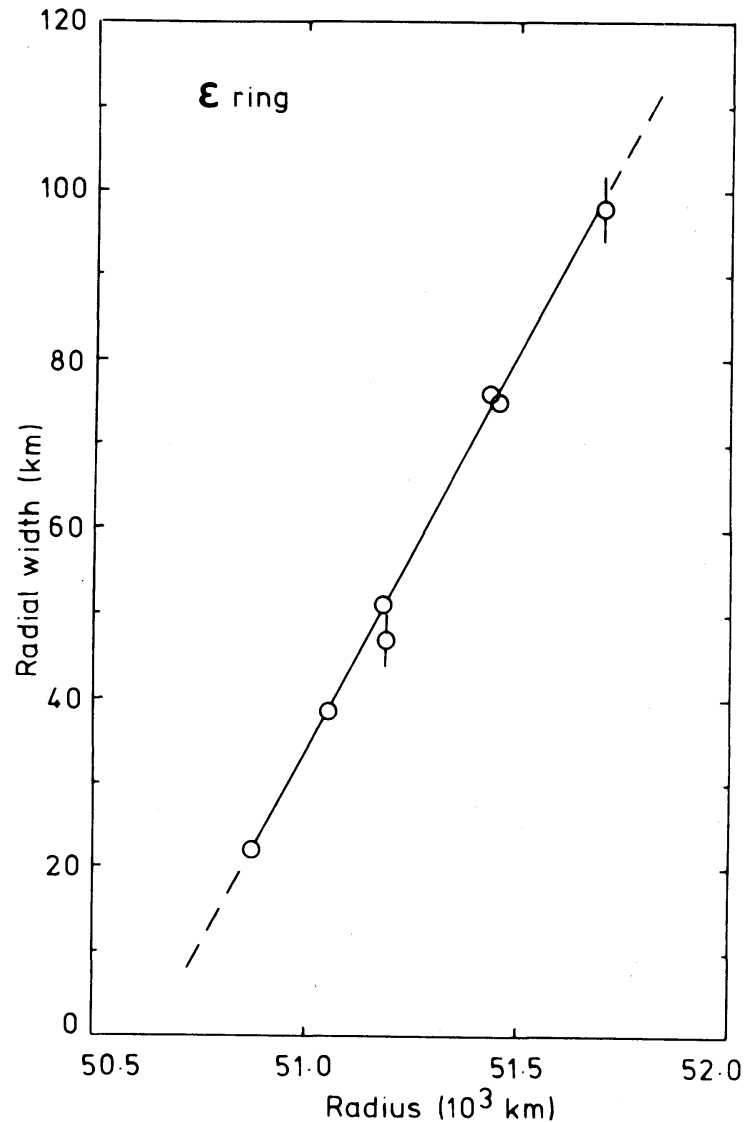

FIG. 2. Observations of radial width and radius (projected onto the satellites' orbital plane) for the $\epsilon$ ring from four stellar occultations, as given in Table V. Except where indicated explicitly, uncertainties in the radial widths are comparable with the diameter of the symbols. Uncertainties in radius are negligible at this scale, except for the 23 December 1977 point, for which the uncertainty may be considerable, but is not known. The line represents a least-squares fit to the data, described in the text.

of the rings, it is important to ensure that there are no systematic differences between the various data sets obtained from different occultations. While the same procedure has been used to reduce data from the occultations of 10 March 1977 (Elliot et al. 1978) and 10 June 1979 (this work), a slightly different and somewhat less accurate procedure was employed in Nicholson et al.'s (1978) analysis of the 10 April 1978 occultation. In addition, the elements of the orbital plane of Uranus's satellites were taken from the Explanatory Supplement to the $\mathrm{AE}$, rather than from the more recent investigation by Dunham (1971).

The data from the 10 April 1978 occultation are therefore reanalyzed here in the manner described in Sec. IV, and with Dunham's (1971) elements for the ring plane. Details of the reanalysis are to be found in the Appendix, and the results in Table VI.

Observations of the 23 December 1977 occultation (Millis and Wasserman 1978) are incomplete, because of the difficult circumstances of the event. It is thus not 
TABLE VI. Revised ring-plane coordinates of the occulting segments, 10 April 1978.

\begin{tabular}{|c|c|c|c|c|}
\hline \multirow[b]{2}{*}{ Ring } & \multicolumn{2}{|c|}{ Immersion } & \multicolumn{2}{|c|}{ Emersion } \\
\hline & $\overline{R(\mathrm{~km})^{\mathrm{a}}}$ & $\theta(\operatorname{deg})^{b}$ & $\overline{R(\mathrm{~km})^{\mathrm{a}}}$ & $\theta(\mathrm{deg})^{\mathrm{b}}$ \\
\hline 6 & 42014 & 53.137 & 42128 & 137.149 \\
\hline 5 & 42381 & 52.587 & 42285 & 137.385 \\
\hline 4 & 42648 & 52.196 & 42679 & 137.963 \\
\hline$\alpha$ & 44833 & 49.262 & 44816 & 140.831 \\
\hline$\beta$ & 45793 & 48.104 & 45805 & 142.026 \\
\hline$\eta$ & 47300 & 46.419 & 47298 & 143.695 \\
\hline$\gamma$ & 47745 & 45.950 & 47746 & 144.166 \\
\hline$\delta$ & 48424 & 45.258 & 48423 & 144.858 \\
\hline$\epsilon$ & 50874 & 42.961 & 51434 & 147.641 \\
\hline
\end{tabular}

a $\pm 2 \mathrm{~km}$.

$\mathrm{b} \pm 0.002$.

possible to reduce these data according to the procedure of Elliot et al. (1978) and of this paper. However, the single observation of the $\epsilon$ ring provided by this event is valuable in constraining possible models of that ring, and for this reason it is retained in two of the solutions to be presented in Sec. $\mathrm{V}$ b.

Data used in the generation of the models presented in Sec. Vb are taken from Table VII in Elliot et al. (1978), Tables IV and VI in the present paper, and from Millis and Wasserman (1978).

\section{b) Elliptical Models}

Nicholson et al. (1978) demonstrated that the observations of radius and azimuth for the $\epsilon$ ring obtained on 10 March 1977, 23 December 1977, and 10 April 1978 were satisfied by a model which consists of a Keplerian ellipse of zero inclination (relative to the satellites' common orbital plane) whose apsidal line precesses at a constant rate of $\sim 1^{\circ} .37 \mathrm{day}^{-1}$. An elliptical rather than an inclined circular model was suggested by the width-radius relation described in Sec. IV b.

The present observations of the $\epsilon$ ring are in rather good agreement with the predictions of this model, viz. radii of 51436 and $51243 \mathrm{~km}$ for immersion and emersion, respectively. The discrepancies between model and data are, however, large enough to warrant a revision of the model.

In addition, the radii of rings $4, \alpha$, and $\beta$ in Table IV provide further confirmation that these three rings are also either noncircular or inclined. Although no information concerning width variations comparable to those shown by the $\epsilon$ ring exists for these rings, it seems reasonable to seek zero-inclination elliptical models for rings $4, \alpha$, and $\beta$ also. It is quite conceivable, however, that inclined circular models could also be obtained for some or all of these three narrow rings.

At the present time, insufficient data exist to meaningfully constrain models for rings 5 and 6 , although these rings also are apparently either noncircular or inclined (Nicholson et al. 1978).

Limits on possible eccentricities of rings $\gamma$ and $\delta$ cannot be obtained directly from present data, since these rings have already been assumed circular with specified radii. [Departures of individual determinations of the radii of these two rings in 1978 and 1979 from the nominal radii of 47746 and $48423 \mathrm{~km}$ lie in the ranges -1 to $+3 \mathrm{~km}(\gamma)$ and -3 to $+1 \mathrm{~km}(\delta)$, comparable with the measurement uncertainties of $\pm 2 \mathrm{~km}$.] An approach which provides some information on the relative departure of $\gamma$ and $\delta$ from circularity, but which cannot distinguish between the two rings, is to adopt the apparent center of one ring (say $\gamma$ ) as coincident with the center of mass of Uranus, and then determine the radii of segments of the other (say $\delta$ ). When this procedure is carried out for the April 1978 and June 1979 occultation data, with the radius of $\gamma$ set at $47746.0 \mathrm{~km}$, the resulting radii for the $\delta$-ring segments are

$$
\begin{array}{ll}
48424.7 \mathrm{~km} & (1978 \mathrm{immersion}), \\
48423.7 \mathrm{~km} & (1978 \text { emersion }), \\
48417.5 \mathrm{~km} & (1979 \text { immersion }), \\
48424.0 \mathrm{~km} & (1979 \text { emersion }),
\end{array}
$$

with measurement uncertainties of $\pm 2 \mathrm{~km}$. The average of these measurements is $48422.5 \mathrm{~km}$, in good agreement with Elliot et al.'s (1978) value of $48423 \mathrm{~km}$, and the standard deviation $\sigma$ is $2.9 \mathrm{~km}$. The latter suggests an upper limit to the eccentricity of $\gamma$ and/or $\delta$ of $\sim \sqrt{2} \sigma / R$ $=8 \times 10^{-5}$, about six times less than the eccentricities obtained below for rings $4, \alpha$, and $\beta$.

For each of the rings $4, \alpha, \beta$, and $\epsilon$, the observed radius $R$, considered as a function of the azimuth $\theta$ and Julian date $t$, is fitted in a least-squares sense by the four-parameter model

$$
R=\bar{R}\left\{1-e \cos \left[\theta-\tilde{\omega}_{0}-\dot{\tilde{\omega}}\left(t-t_{0}\right)\right]\right\},
$$

with $\bar{R}$ the mean radius, $e$ the eccentricity, $\tilde{\omega}_{0}$ the azimuth of periapse at epoch $t_{0}$, and $\dot{\tilde{\omega}}$ the apsidal precession rate. The standard epoch, $t_{0}$, is defined as $2000 \mathrm{UT}$, 10 March $1977=$ JD 2443213.33. Correct to second order in $e, \bar{R}=a\left(1-e^{2} / 2\right)$, where $a$ is the semimajor axis, but only for the $\epsilon$ ring does $\bar{R}$ differ measurably from $a$. Solutions are obtained for a range of initial estimates of the precession rate $\dot{\tilde{\omega}}$.

In general, the values of $a, e$, and $\tilde{\omega}_{0}$ are well defined for each ring, but a family of solutions exists with values of $\dot{\tilde{\omega}}$ separated approximately by integer multiples of $0.877 \mathrm{day}^{-1}$. This property of the solutions is an artifact of the nearly equal spacing in time of the three important occultations, viz. 395 and 426 days. An increase of 0.877 day $^{-1}$ in $\dot{\tilde{\omega}}$ corresponds to one extra complete revolution of the apsidal line in 411 days. In the case of the $\epsilon$ ring, the existence of the 23 December 1977 point eliminates from consideration all but one member of this family, unless extremely high precession rates $\left(>10^{\circ} \mathrm{day}^{-1}\right)$ are allowed. If it is assumed that the oblateness of Uranus is largely responsible for the apsidal precession of the rings (see Sec. VI), then the expected precession rates for rings $4, \alpha$, and $\beta$ may be estimated by scaling the $\epsilon$ 
TABLE VII. Elliptical models of the rings.

\begin{tabular}{|c|c|c|c|c|c|}
\hline Model & $a(\mathrm{~km})$ & $e \times 10^{4}$ & $\tilde{\omega}_{0}$ & $\dot{\tilde{\omega}}\left(\right.$ deg day $\left.^{-1}\right)$ & $\begin{array}{c}\text { rms } \\
\text { deviation } \\
(\mathrm{km})\end{array}$ \\
\hline $4 a$ & $42693 \pm 13$ & $11.9 \pm 4.3$ & $124^{\circ} \pm 11^{\circ}$ & $2.590 \pm 0.032$ & 3.0 \\
\hline $4 b$ & $42668.7 \pm 1.0$ & $5.99 \pm 0.34$ & $167^{\circ} 0 \pm 5^{\circ} .0$ & $2.366 \pm 0.011$ & 1.0 \\
\hline$\alpha$ & $44839.1 \pm 1.0$ & $6.02 \pm 0.28$ & $325^{\circ} 4 \pm 3.5$ & $2.232 \pm 0.015$ & 2.1 \\
\hline$\beta$ & $45784.2 \pm 1.8$ & $4.89 \pm 0.53$ & $227^{\circ} 0 \pm 8.1$ & $2.022 \pm 0.014$ & 3.0 \\
\hline$\epsilon_{11}$ & $51276.6 \pm 9.3$ & $82.9 \pm 2.4$ & $216^{\circ} 7 \pm 2.8$ & $1.3621 \pm 0.0048$ & 19.8 \\
\hline$\epsilon_{9}$ & $51272.6 \pm 3.8$ & $79.3 \pm 1.1$ & $215^{\circ} 3 \pm 1.2$ & $1.3630 \pm 0.0020$ & $7.4^{\mathrm{a}}$ \\
\hline$\epsilon_{8}$ & $51271.1 \pm 2.1$ & $79.1 \pm 0.6$ & $214^{\circ} 5 \pm 0.7$ & $1.3634 \pm 0.0011$ & 3.8 \\
\hline$\alpha_{78}$ & $44839 \pm .1$ & $6.3 \pm 0.3$ & $325^{\circ} \pm 2^{\circ}$ & 2.20 (fixed) & $1.6^{\mathrm{b}}$ \\
\hline$\epsilon_{78}$ & $51284 \pm 6$ & $78.0 \pm 1.2$ & $212^{\circ} \pm 2^{\circ}$ & $1.374 \pm 0.006$ & $7^{b}$ \\
\hline
\end{tabular}

a Preferred $\epsilon$ model.

b Nicholson et al. (1978).

rate as $a^{-7 / 2}$. In each case, this expected rate is quite close to that of one member of the family of solutions, and this member is chosen as our preferred model for that ring.

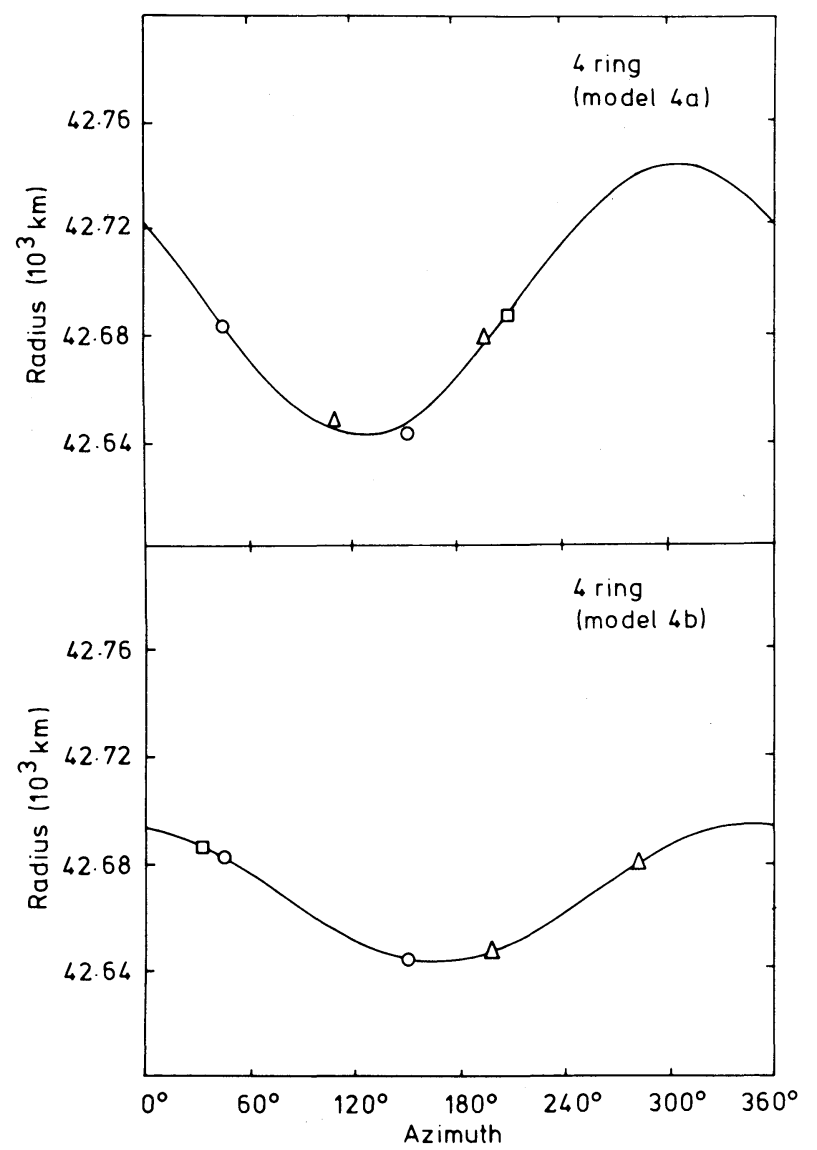

FIG. 3. Two possible elliptical models for ring $4,4 \mathrm{a}$ and $4 \mathrm{~b}$, compared with the occultation data available. Parameters for the models are given in Table VII. Azimuths of the data points have been corrected to the standard epoch $t_{0}$ (see text), by removal of the intervening apsidal precession predicted by the model:

$$
\theta\left(t_{0}\right)=\theta(t)-\dot{\tilde{\omega}}\left(t-t_{0}\right) .
$$

Data from the different stellar occultations are indicated by circles (10 March 1977), triangles (10 April 1978), and squares (10 June 1979).
In Table VII, the elements of elliptical models for each of the rings $4, \alpha, \beta$, and $\epsilon$, chosen as described above, are presented. In addition, the rms deviation of the data from each of the models is given. These models are also presented in Figs. 3-6 as plots of radius versus azimuth, along with the data reduced to the standard epoch $t_{0}$ by the removal of apsidal precession.

For ring 4, two quite distinct models are possible (each belonging to a different family of solutions) with the present five data points. The apsidal precession rate of model $4 \mathrm{a}$ is closer to the expected rate for this ring of $2^{\circ} .587 \mathrm{day}^{-1}$, but the rms deviation of the data is smaller for model $4 \mathrm{~b}$. Future occultation observations of this ring should resolve this ambiguity.

Models for rings $\alpha$ and $\beta$ are rather well defined by the ten data points ( 6 from 10 March 1977) available for each ring. The rms deviations are comparable to, or smaller than, likely random errors in the data due to timing errors. The precession rate for $\beta$ is in excellent agreement with the expected $2^{\circ} .026 \mathrm{day}^{-1}$, but the rate for $\alpha$ differs by $\sim 3.5 \sigma$ from its expected value of $2^{\circ} .179$ $\mathrm{day}^{-1}$. A model for the $\alpha$ ring with the precession rate fixed at $2^{\circ} .179$ day $^{-1}$ provides a good fit to all data points except for the 10 June 1979 immersion datum, which

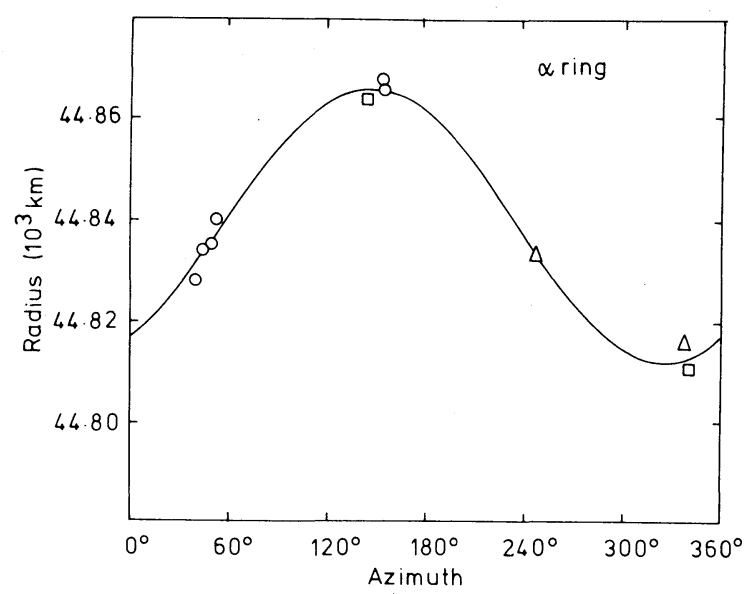

Fig. 4. The elliptical model for ring $\alpha$ compared with data corrected to the standard epoch. Symbols are as in Fig. 3. Note the expansion of the radial scale in comparison with that of Fig. 3. 


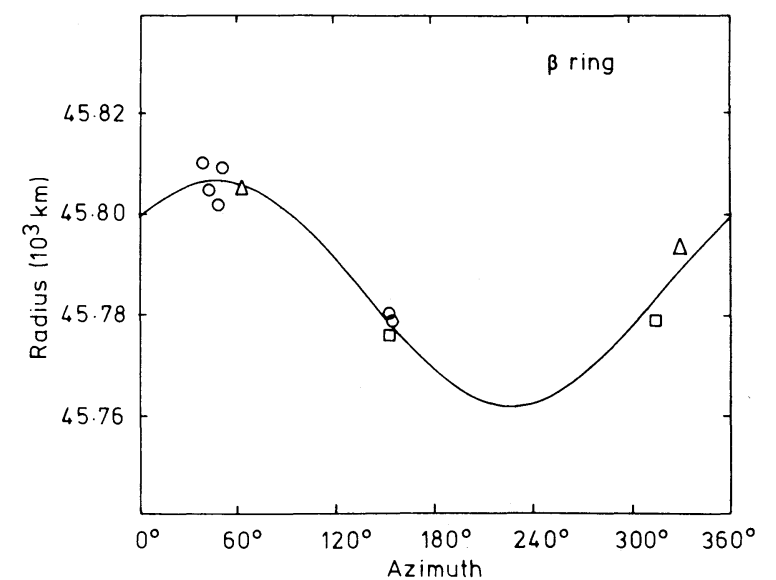

FIG. 5. The elliptical model for ring $\beta$ compared with data corrected to the standard epoch. Symbols are as in Fig. 3. Radial scale is the same as that of Fig. 4.

falls $12 \mathrm{~km}$ below the model radius. Such a discrepancy is equivalent to a timing error of $+0.6 \mathrm{~s}$, which might conceivably be produced by noise in the data. The elements of this model are

$$
\begin{gathered}
a=44840.7 \pm 2.0 \mathrm{~km}, \\
e=(6.14 \pm 0.63) \times 10^{-4}, \\
\tilde{\omega}_{0}=335^{\circ} .4 \pm 6.3
\end{gathered}
$$

and do not differ greatly from those of the model presented in Table VII. The rms deviation of the data from the model is $4.5 \mathrm{~km}$.

For the $\epsilon$ ring, three models are presented, labeled $\epsilon_{11}$, $\epsilon_{9}$, and $\epsilon_{8}$, with the subscripts denoting the number of data points used in the least-squares solution. In the first solution, all available observations of the $\epsilon$ ring are included, six of which were made during the 10 March 1977 occultation. Since these six observations essentially represent only two segments of the ring, they are equivalent to only two independent data points. For solution $\epsilon_{9}$, two of these six points (Kavalur and Peking observations) are omitted, because they appear to be inconsistent with the KAO and Perth observations of the same event. Possible uncertainties in these omitted observations are discussed by Nicholson et al. (1978). Standard errors in the fitted parameters and the rms deviation of the data are considerably smaller for solution $\epsilon_{9}$ than for $\epsilon_{11}$, and this is our preferred model for the $\epsilon$ ring. A solution is also presented in Table VII $\left(\epsilon_{8}\right)$ for which the 23 December 1977 point has been omitted, as well as the two points referred to above. This solution exhibits even smaller standard errors and rms deviation, but the values of the parameters themselves do not differ significantly from those of solution $\epsilon_{9}$.

Comparison of the present model for the $\epsilon$ ring (i.e., solution $\epsilon_{9}$ ) with that of Nicholson et al. (1978), listed as $\epsilon_{78}$ in Table VII, shows the two models to differ in each of the elements by no more than twice the standard error of the 1978 elements. Note that the uncertainty in the apsidal precession rate has decreased by a factor of 3 relative to the 1978 model. Nicholson et al. (1978) also presented a tentative elliptical model of the $\alpha$ ring, with the apsidal precession rate fixed at $2.20 \mathrm{day}^{-1}$. The elements of this model ( $\alpha_{78}$ in Table VII) are also in good agreement with those of the present model. Elliot et al. (1978) made rough estimates of possible eccentricities for rings $\alpha$ and $\beta$ of $(10.9 \pm 2.5) \times 10^{-4}$ and $\left(3.7_{-2.6}^{+5.2}\right)$ $\times 10^{-4}$, respectively.

\section{URANUS'S GRAVITATIONAL MOMENTS}

Apsidal precession of the orbits of the particles which make up the rings of Uranus is due to at least two effects. The first and by far the most important of these is the secular precession caused by the oblate figure of the planet. For an orbit of zero inclination to the equatorial plane (i.e., to the satellites' common orbital plane), this precession is given, to $O(e)$ and $O\left(J_{2}^{2}\right)$, by

$$
\dot{\tilde{\omega}}_{P}=\frac{(G M)^{1 / 2} R^{2}}{a^{7 / 2}}\left[(3 / 2) J_{2}-(15 / 4) J_{4}(R / a)^{2}\right]
$$

(Brouwer and Clemence 1961). In this expression, $G$ is the gravitational constant, $M$ is the mass of Uranus, $R$ is the equatorial radius of Uranus, and $J_{2}$ and $J_{4}$ are the usual dimensionless coefficients in the spherical harmonic expansion of a planet's gravitational field.

The second contribution to apsidal precession is due to the five satellites of Uranus, principally to Ariel (U1), and is given approximately by

$$
\dot{\tilde{\omega}}_{s} \simeq \frac{3}{4}\left(\frac{G M}{a^{3}}\right)^{1 / 2} \sum_{i=1}^{5} \frac{m_{i}}{M}\left(\frac{a}{a_{i}}\right)^{3}
$$

where $m_{i}$ and $a_{i}$ are the mass and orbital semimajor axis of the $i$ th satellite. With rather uncertain values for the

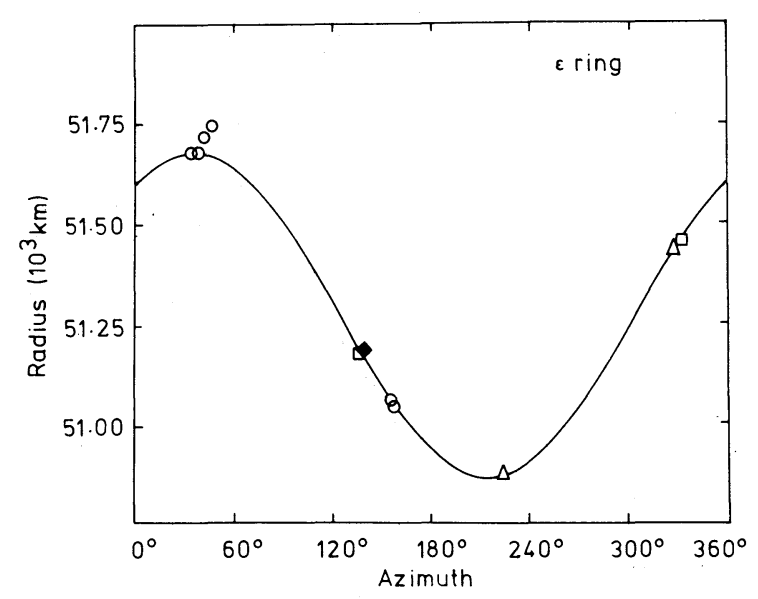

FIG. 6. Elliptical model $\epsilon_{9}$ for the $\epsilon$ ring, compared with data corrected to the standard epoch. The observation of 23 December 1977 is represented by a diamond, while the other symbols are as in Fig. 3. Two of the 10 March 1977 points are not included in this fit to the data (see text), but are shown in the figure. Note the compression of the radial scale by a factor of 12.5 in comparison with that of Figs. 4 and 5. 
TABLE VIII. Values of $\Psi=J_{2}-5 / 2 J_{4}(R / a)^{2}$ obtained from the apsidal precession rates of elliptical ring models.

\begin{tabular}{cc}
\hline Ring model & $\Psi \times 10^{3}$ \\
\hline $4 \mathrm{a}$ & $3.396 \pm 0.042$ \\
$4 \mathrm{~b}$ & $3.096 \pm 0.014$ \\
$\alpha$ & $3.474 \pm 0.023$ \\
$\beta$ & $3.385 \pm 0.023$ \\
$\epsilon_{9}$ & $3.390 \pm 0.005$ \\
\hline
\end{tabular}

masses of the satellites given by Greenberg (1975), which are based on a dynamical analysis of the system by Dunham, $\dot{\tilde{\omega}}_{s}$ varies from $1.0 \times 10^{-3} \mathrm{deg} \mathrm{day}^{-1}$ for ring 4 to $1.3 \times 10^{-3} \mathrm{deg} \mathrm{day}^{-1}$ for ring $\epsilon$. Only for the $\epsilon$ ring is this contribution even comparable to the present uncertainty in the ring precession rate.

Contributions to the apsidal precession of ring particles by the gravitational action of the sun are $\sim 10^{-7}$ deg day ${ }^{-1}$, and quite negligible. Further contributions could arise from eccentricity-type resonant interactions between the ring particles and one or more satellites, but no such interactions have yet been demonstrated.

It is assumed here that the apsidal precession of the rings, as determined from the models of Sec. V, represents the average precession rate of the particles which make up the rings. Observations of the $\epsilon$ ring over a period of over two years indicate that differential precession across this ring, due to the strong dependence of $\dot{\tilde{\omega}}_{p}$ on $a$, is suppressed. Two possible mechanisms for this suppression have been advanced. Goldreich and Tremaine (1979) have demonstrated that, given a sufficient surface density $\left(\sim 25 \mathrm{~g} \mathrm{~cm}^{-2}\right.$ for the $\epsilon$ ring), the self-gravity of the ring can act to prevent differential precession of the orbits of the ring particles. A second process suggested by the same authors involves collisions between ring particles which attempt to precess differentially, and

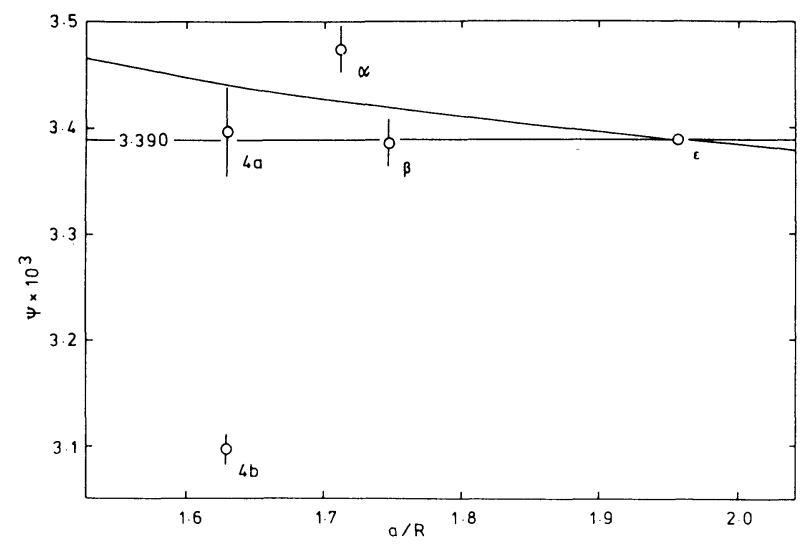

FIG. 7. The quantity $\Psi \equiv J_{2}-5 / 2 J_{4}(R / a)^{2}$, as determined from the apsidal precession rates for the elliptical ring models, plotted as a function of normalized ring semimajor axis, $a / R$. Values of $\Psi$ for each of the two possible models for ring 4 are shown. The error bar on $\Psi_{\epsilon}$ is comparable to the diameter of the symbol. The horizontal line represents our preferred best estimate of the gravitational moments: $J_{2}$ $=3.390 \times 10^{-3}, J_{4}=0.0$; the sloping curve represents the least-squares fit described in the text.
TABLE IX. Gravitational moments for the other Jovian planets.

\begin{tabular}{lcccc}
\hline & $\begin{array}{c}\text { Equatorial } \\
\text { Planet }\end{array}$ & & & \\
radius $(\mathrm{km})$ & $J_{2} \times 10^{3}$ & $J_{4} \times 10^{3}$ & $J_{4} /\left(J_{2}\right)^{2}$ \\
\hline Jupiter & 71398 & $14.75 \pm 0.05^{\mathrm{a}}$ & $-0.58 \pm 0.04^{\mathrm{a}}$ & -2.7 \\
Saturn & 59671 & $16.67 \pm 0.02^{\mathrm{b}}$ & $-1.03 \pm 0.07^{\mathrm{b}}$ & -3.7 \\
Neptune & 24753 & $4.00 \pm 0.40^{\mathrm{c}}$ & $-0.039^{\mathrm{d}}$ & -2.4 \\
\hline \hline
\end{tabular}

a Null et al. (1975).

b Brouwer and Clemence (1961)

c Brouwer and Clemence (1961), corrected to the radius determined by Freeman and Lyngå (1970).

d Gavrilov and Zharkov (1977), theoretical estimate.

may result in something analogous to a shock which impulsively transfers momentum to the ring particles. A model for the rings advanced by Dermott et al. (1979) also suggests that differential precession should not occur. It seems reasonable, therefore, to associate the "observed" precession rates of the rings with their average semimajor axes, as listed in Table VII.

From the values of $a$ and $\dot{\tilde{\omega}}$ in Table VII, after the small correction for satellite-induced precession $\dot{\tilde{\omega}}_{s}$ is applied, the dimensionless quantity

$$
\Psi \equiv J_{2}-5 / 2 J_{4}(R / a)^{2}
$$

is calculated for each of rings 4 (models $4 \mathrm{a}$ and $4 \mathrm{~b}$ ), $\alpha$, $\beta$, and $\epsilon\left(\operatorname{model} \epsilon_{9}\right)$, and listed in Table VIII. In Fig. 7, $\Psi$ is plotted as a function of $a / R$. Values adopted for the mass and radius of Uranus are

$$
G M=5.78418 \times 10^{6} \mathrm{~km}^{3} \mathrm{~s}^{-2},
$$

based on Dunham's (1971) determination of $M_{\odot} / M=$ $22945 \pm 15$, and

$$
R=26200 \mathrm{~km}
$$

\section{(J. L. Elliot 1979, private communication).}

Clearly, it is impossible to choose values of $J_{2}$ and $J_{4}$ which will adequately fit the observations for all four rings, irrespective of which model is accepted for ring 4. A determination of these two quantities based on a weighted least-squares fit to $\Psi_{4 \mathrm{a}}, \Psi_{\alpha}, \Psi_{\beta}$, and $\Psi_{\epsilon}$ gives

$$
\begin{aligned}
& J_{2}=(3.27 \pm 0.05) \times 10^{-3}, \\
& J_{4}=(-1.8 \pm 0.8) \times 10^{-4},
\end{aligned}
$$

with a correlation coefficient of 0.996 . This fit is shown in Fig. 7, but is unacceptable for two reasons. First, only one point $(\epsilon)$ lies within one sigma of the fitted relation $\Psi(a / R)$. Second, the absolute value of $J_{4}$ obtained is unreasonably large, when compared with determinations of this quantity for the other Jovian planets.

Such a comparison is most readily made via the ratio $J_{4} /\left(J_{2}\right)^{2}$, which is, to first order, independent of the rotation rate of the planet and, independent of the adopted equatorial radius. Table IX lists values of $J_{2}, J_{4}$, and $J_{4} /\left(J_{2}\right)^{2}$ for Jupiter, Saturn, and Neptune. (For Neptune, $J_{4}$ has not been observationally determined, and is taken from a theoretical calculation by Gavrilov 
and Zharkov 1977.) All three of these planets have -4 $<J_{4} /\left(J_{2}\right)^{2}<-2$, which suggests that, for Uranus, $J_{4}$ should lie in the range $(-4.6$ to -2.3$) \times 10^{-5}$, or be four to eight times smaller in absolute magnitude than the least-squares estimate given above.

An alternative procedure is to disregard the apparently aberrant value of $\Psi_{\alpha}$, and consider only the data for the three other rings. If model $4 \mathrm{a}$ is adopted for ring 4 , then the remaining data are consistent with

$$
J_{2}=(3.390 \pm 0.005) \times 10^{-3}
$$

and

$$
J_{4}=0.0 \text {. }
$$

An upper limit of $\left|J_{4}\right| \lesssim 1 \times 10^{-4}$ (one sigma), which accommodates the probable range given above, is set by the uncertainties in the present determinations of $\Psi_{4 a}$ and $\Psi_{\beta}$, but is not reflected in the quoted uncertainty for $J_{2}$. Any future determination of $J_{4}$ would require a corresponding modification to our estimate for $J_{2}$ according to

$$
\partial J_{2} / \partial J_{4}=5 / 2\left(R / a_{\epsilon}\right)^{2}=0.6528 .
$$

This estimate for $J_{2}$ improves upon that of Nicholson et al. $(1978),(3.43 \pm 0.02) \times 10^{-3}$, which was also obtained on the (unstated) assumption that $J_{4}=0.0$.

\section{CONCLUSIONS}

(1) Occultations by Uranus and six of its nine known rings $(4, \alpha, \beta, \gamma, \delta$, and $\epsilon)$ were observed at Las Campanas on 10 June 1979.

(2) Observations of rings $\gamma$ and $\delta$ are consistent with the hypothesis that they are coplanar, circular, and concentric. An approximate upper limit of $8 \times 10^{-5}$ is obtained for the eccentricity of $\gamma$ and/or $\delta$. Corrections to the relative positions of Uranus and the occulted star are based on Elliot et al.'s (1978) determination of the radii of these two rings.

(3) The width-radius relation established previously for the $\epsilon$ ring by Nicholson et al. (1978) is confirmed.

(4) Data obtained for the $\epsilon$ ring are in accord with the predictions of the elliptical model of Nicholson et al., and permit a refinement of that model. The apsidal precession rate is found to be $1^{\circ} .363 \pm 0.002 \mathrm{day}^{-1}$, and the eccentricity is $(7.9 \pm 0.1) \times 10^{-3}$.

(5) Similar, zero-inclination (with respect to the satellites' common orbital plane), elliptical models are presented for rings $\alpha$ and $\beta$, with eccentricities of (6.0 $\pm 0.3) \times 10^{-4}$ and $(4.9 \pm 0.5) \times 10^{-4}$, respectively. Two possible elliptical models are obtained for ring 4 , with eccentricities of $(1.2 \pm 0.4) \times 10^{-3}$ and $(6.0 \pm 0.3) \times$ $10^{-4}$. Present data are insufficient to constrain similar models for rings 5 and 6 .

(6) Apsidal precession rates for rings 4 (model 4a), $\beta$, and $\epsilon$, but not $\alpha$, are consistent with planetary gravitational moments of $J_{2}=(3.390 \pm 0.005) \times 10^{-3}$ and $J_{4}=0.0$, although values of $\left|J_{4}\right| \lesssim 1 \times 10^{-4}$ cannot be
TABLE X. Parameters used in the reanalysis of the 10 April 1978 occultation.

\begin{tabular}{ll}
\hline \multicolumn{1}{c}{ Parameter } & \multicolumn{1}{c}{ Value } \\
\hline Greenwich sidereal time at $0^{\mathrm{h}} \mathrm{ET}$ & $13^{\mathrm{h}} 10^{\mathrm{m}} 40^{\mathrm{s}} .0$ \\
$\Delta T=$ ET $-\mathrm{UT}(\mathrm{s})$ & 48.7 \\
Julian date at $0^{\mathrm{h}} \mathrm{UT}$, & 2443608.5 \\
Elements of satellites' orbital plane (equinox of & \\
date): & $74^{\circ} .925$ \\
$J$ & $167^{\circ} .125$ \\
$N$ & \\
Auxiliary angles: & $57^{\circ} .203$ \\
$B$ & $273^{\circ} .019$ \\
$P$ & $353^{\circ} .623$ \\
$U$ & \\
\hline \hline
\end{tabular}

excluded. Resolution of the apparent discrepancy in the $\alpha$ precession rate would require a 0.6 -s error in the 1979 immersion datum.

Observations of another occultation by rings $\alpha-\epsilon$ on 20 March 1980 have recently been reported by Elliot $e t$ al. (1981), who constructed elliptical models for rings $4, \alpha, \beta$, and $\epsilon$ based on the same assumptions concerning the radii of rings $\gamma$ and $\delta$ as are made in the present work. These models, which incorporate data from the 10 June 1979 occultation, are in close agreement with the models presented in Table VII above, the only significant deviation being in the eccentricity of ring $\alpha$, for which a value of $(7.0 \pm 0.4) \times 10^{-4}$ is obtained. The model for ring 4 is essentially equivalent to our model $4 \mathrm{a}$. Because the precession rates of the four rings were constrained to be consistent with unique values of $J_{2}$ and $J_{4}$, the problem of the aberrant $\alpha$ precession rate is not specifically addressed by Elliot $e t$ al.

The authors gratefully acknowledge the assistance of F. Peralta, W. Robinson, L. Papic, and J. Mould in making the observations at Las Campanas. J. L. Elliot kindly provided a preprint concerning the 20 March 1980 occultation. This work was supported in part by NASA.

\section{APPENDIX: REANALYSIS OF THE 10 APRIL 1978 RING OCCULTATIONS}

For the reasons of consistency outlined in Sec. $\mathrm{V}$ a, it is desirable that data from the ring occultations of 10 April 1978, as reported by Nicholson et al. (1978), be reanalyzed in accordance with the procedure described in Sec. IV of this paper. Included in this revision is the substitution of Dunham's (1971) elements for the orbital plane of Uranus's satellites for those given in the Explanatory Supplement to the AE and originally obtained by $\mathrm{H}$. Struve in 1913. This substitution amounts to a shift of 0.123 in the direction of the normal to the orbital plane.

Values for parameters used in the reanalysis are given in Table X. Observatory coordinates are the same as for the 10 June 1979 occultation, and may be found in Table II. Observations of the $\gamma$ and $\delta$ rings are again used 
separately to estimate the apparent offset of the center of mass of Uranus from its ephemeris position:

$$
\begin{aligned}
(\Delta \xi, \Delta \eta)_{\gamma} & =(-9277.0 \pm 1.4,-6143.2 \pm 1.9), \\
(\Delta \xi, \Delta \eta)_{\delta} & =\left(-9277.2 \pm 1.4,-6141.2^{\circ} \pm 1.9\right),
\end{aligned}
$$

with all dimensions in kilometers. The consistency of these determinations is quite impressive, and adds further support to the hypothesis that these two rings are circular and concentric. The adopted mean offset is

$$
\Delta \xi=-9277.1 \mathrm{~km}
$$

$$
\Delta \eta=-6142.2 \mathrm{~km},
$$

equivalent to -0.722 arcsec in right ascension and -0.478 arcsec in declination, and again reflects errors in both the ephemeris of the planet and the stellar position, as given by Klemola and Marsden (1977).

Final results of the reanalysis, in the form of ringplane coordinates for the 18 occulting segments of the rings, are given in Table VI above. Differences between these results and those obtained by Nicholson et al. (1978) lie in the ranges $-7 \mathrm{~km}(\epsilon$ immersion) to $+12 \mathrm{~km}$ (ring 6 immersion) in radius, and $+0^{\circ} 02$ to $+0^{\circ} 06$ in aximuth.

\section{REFERENCES}

Brouwer, D., and Clemence, G. M. (1961). In Planets and Satellites, The Solar System, edited by G. P. Kuiper and B. M. Middlehurst (University of Chicago, Chicago), Vol. III, Chap. 3.

Dermott, S. F., Gold, T., and Sinclair, A. T. (1979). Astron. J. 84, 1225.

Dunham, D. W. (1971). Ph.D. thesis, Yale University.

Elliot, J. L., Dunham, E., Wasserman, L. H., Millis, R. L., and Churms, J. (1978). Astron. J. 83, 980.

Elliot, J. L., Frogel, J. A., Elias, J. H., Glass, I. S., French, R. G., Mink, D., and Liller, W. (1981). Astron. J. 86, 127.

Freeman, K. C., and Lyngå, G. (1970). Astrophys. J. 160, 767.

Gavrilov, S. V., and Zharkov, V. N. (1977). Icarus 32, 443.

Goldreich, P., and Tremaine, S. (1979). Astron. J. 84, 1638.
Greenberg, R. (1975). Icarus 24, 325.

Klemola, A. R., and Marsden, B. G. (1977). Astron. J. 82, 849.

Liller, W. (1977). Astron. J. 82, 929.

Millis, R. L., and Wasserman, L. H. (1978). Astron. J. 83, 993.

Millis, R. L., Wasserman, L. H., and Birch, P. V. (1977). Nature 267, 330.

Nicholson, P. D., Persson, S. E., Matthews, K., Goldreich, P., and Neugebauer, G. (1978). Astron. J. 83, 1240.

Null, G. W., Anderson, J. D., and Wong, S. K. (1975). Science 188, 476.

Smart, W. M. (1977). Textbook on Spherical Astronomy, 6th ed., revised by R. M. Green (Cambridge University, Cambridge). 